\title{
BRIEF PAPER \\ A New Common-Mode Stabilization Method for a CMOS Cascode Class-E Power Amplifier with Driver Stage
}

\author{
Zhisheng LI ${ }^{\dagger \text { a) }}$, Nonmember, Johan BAUWELINCK ${ }^{\dagger}$, Member, Guy TORFS ${ }^{\dagger}$, Xin YIN ${ }^{\dagger}$, \\ and Jan VANDEWEGE ${ }^{\dagger}$, Nonmembers
}

\begin{abstract}
SUMMARY This paper presents a new common-mode stabilization method for a CMOS differential cascode Class-E power amplifier with LCtank based driver stage. The stabilization method is based on the identification of the poles and zeros of the closed-loop transfer function at a critical node. By adding a series resistor at the common-gate node of the cascode transistor, the right-half-plane poles are moved to the left half plane, improving the common-mode stability. The simulation results show that the new method is an effective way to stabilize the PA.

key words: CMOS, Class-E power amplifiers, common mode, stability, closed-loop transfer function, pole, zero, right half plane
\end{abstract}

\section{Introduction}

In the lower $\mathrm{GHz}$ range, more and more low output power power amplifiers (PAs) are implemented in CMOS for cost reduction or for promoting monolithic integration. Stability is an important design goal in the design of PAs. Many papers [1]-[4] have been published to analyze the largesignal stability problem. The differential cascode topology is widely used in deep sub-micron CMOS PAs due to the low breakdown voltage of the CMOS technology. However, a differential circuit can run into the common-mode stability problem. [5] analyzes the common-mode stability of a single stage PA, but without driver stage nor cascode transistors. In this paper, we will show that the cascode transistors provide a new means to stabilize common-mode instabilities by adding a common-gate resistor. This paper presents a common-mode stability analysis of a CMOS differential cascode Class-E PA [6] with LC tank-load driver stage based on the analysis of poles and zeros of the closed-loop transfer function at a critical node.

This paper is organized as follows. Section 2 introduces the theoretical background and models used for the analysis of the common-mode stability. Section 3 presents numerical results of a PA, followed by a discussion of a new stabilization method. Section 4 gives simulation results to verify the stabilization method. Finally, conclusions are drawn in Sect. 5.

\section{Common-Mode Stability Analysis}

A simplified topology of the differential cascode Class-E

\footnotetext{
Manuscript received November 3, 2011.

$\dagger$ The authors are with the Department of Information Technology of Ghent University/IMEC, Belgium.

a) E-mail: zhisheng.li@intec.ugent.be

DOI: 10.1587/transele.E95.C.765
}

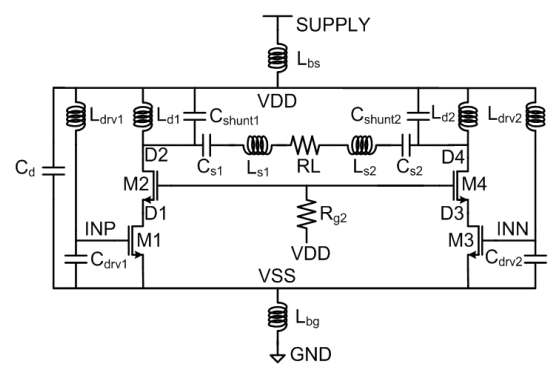

Fig. 1 Topology of the differential cascode Class-E PA with output impedance of the driver.

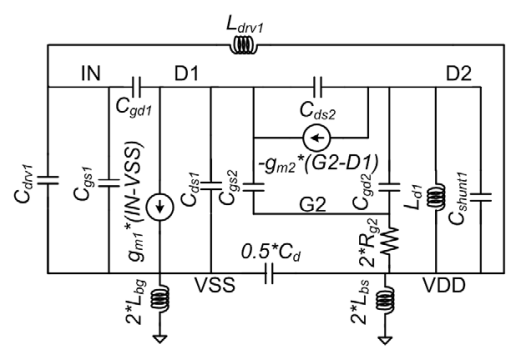

Fig. 2 Common-mode small signal circuit of half the circuit.

PA including the output impedance of the driver is shown in Fig. 1, in which $R_{L}$ is the effective load resistance, and $L_{b s}$ and $L_{b q}$ are the bondwire inductances of the supply and ground respectively. $C_{d}$ is the on-chip decoupling capacitance. The output impedance of the driver includes the drain inductances ( $L_{d r v 1}$ and $L_{d r v 2}$ ) with parasitic resistances $R_{l d r v 1}$ and $R_{l d r v 2}$ respectively, and the output capacitances $\left(C_{d r v 1}\right.$ and $C_{d r v 2}$ ) of the driver. Other parts of the driver are not shown for simplicity. Figure 2 shows the common-mode equivalent small-signal circuit. The common-mode stability has been analyzed in [5], but without taking into account the cascode transistors or a driver stage. The common-mode equivalent circuit is without the output series resonant paths, as both outputs carry identical signals in common mode. In common mode, the bond-wire inductances $L_{b g}$ and $L_{b s}$ and the decoupling capacitance $C_{d}$ must be considered.

As proposed in [7], except for exact pole-zero cancellations, all the closed-loop transfer functions at different nodes share the same characteristic equation, which means they have the same poles. So, the need for initial consideration of different observation nodes arises. In this paper, the closed-loop transfer function at node IN is used to an- 
alyze the stability, starting from the matrix equations based on Kirchhoff's current and voltage Laws:

$$
Y_{c m} \times V_{c m}=I_{c m}
$$

in which, $Y_{c m}$ is the common-mode admittance matrix and $V_{c m}$ represents the common-mode network voltages (at nodes IN, D1, D2, G2, VDD and VSS) corresponding to the common-mode currents injected at the same nodes, defined in $I_{c m}$. The $Y_{c m}$ is given in (2), in which

$$
Y_{d}=s C_{\text {shunt } 1}+1 /\left(s L_{d 1}\right)
$$

Consider a unit current entering node IN, $I_{c m}$ and the corresponding $V_{c m}$ can be written as:

$$
\begin{aligned}
I_{c m} & =\left[\begin{array}{llllll}
1 & 0 & 0 & 0 & 0 & 0
\end{array}\right]^{T} \\
V_{c m} & =\left[\begin{array}{llllll}
I N & D 1 & D 2 & G 2 & V D D & V S S
\end{array}\right]^{T}
\end{aligned}
$$

By solving (1) for $I_{c m}$ and $V_{c m}$ defined in (4) and (5), the common-mode closed-loop transfer function $H_{c l, c m}(s)$ at IN is found as $I N$ (divided by $1 \mathrm{~A}$ ) in $V_{c m}$, which can be calculated numerically. With the obtained $H_{c l, c m}(s)$, the respective poles and zeros can be easily extracted using math tools, e.g. Matlab in our case. No analytical expressions of poles or zeros are required in this way. The root locus can be simply obtained by sweeping the parameters we want to track.

\section{Stabilization Methodology}

A typical pole-zero map of the common-mode closed-loop transfer function $H_{c l, c m}$ at IN is shown in Fig. 3 for a typical system. A pair of right-half-plane (RHP) complexconjugate poles is not shown in Fig. 3 because the absolute value of their imaginary parts is much higher than the characteristic frequency of CMOS (more than $10^{16} \mathrm{~Hz}$ in our case). Thus the modeling of these two poles is not accurate, and the poles are neglected. However, there exists another pair of RHP complex-conjugate poles in Fig. 3, which implies common-mode instability. This pair must be moved to the left half plane (LHP).

For the common-mode stability analysis, all the design parameters in the core circuit are not altered because these should be optimized for the differential-mode operation, so only $L_{b q}, L_{b s}$ and $C_{d}$ are considered here as degrees of freedom. To make the results more clear, only the evolution of

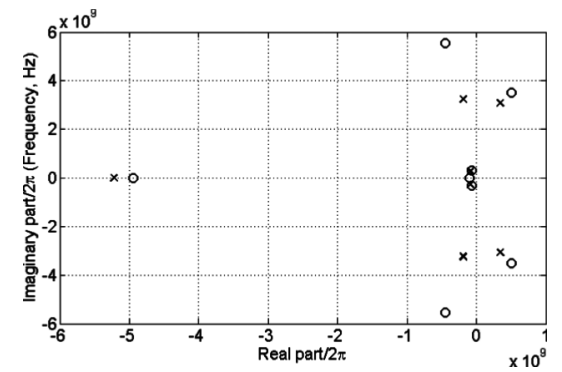

Fig. 3 Pole-zero map of $H_{c l, c m}$ at IN in common mode. the real part of the RHP poles as a function of $L_{b g}, L_{b s}$ and $C_{d}$ is shown in Fig. 4(a). As shown in Fig. 4(a), the effect of $L_{b q}$ and $L_{b s}$ on the RHP poles of $H_{c l, c m}$ can be neglected. As $C_{d}$ increases, the RHP conjugate poles are moving towards the LHP but are still in the RHP. A resistor can be added in series with $C_{d}$ to move the RHP poles to the LHP. This has been analyzed in detail in [5], and won't be analyzed here. However, adding a resistor in series with $C_{d}$ degrades the decoupling performance. Here a new method to stabilize the circuit in common mode is proposed, which doesn't degrade the circuit performance. This new method is based on adding a series resistor $R_{g 2}$ at the gates of the cascode transistors (M2, M4 in Fig. 1).

Due to the existence of multiple feed-back loops in common mode, it is very difficult to get a simple expression to describe why $R_{g 2}$ improves the stability. However, we can simply consider two extreme conditions: $R_{g 2}$ being 0 or infinite. Figure 4(b) shows the real part of the RHP poles as a function of $L_{d r v 1}$ when $R_{g 2}$ is 0 and infinite. When $R_{g 2}$ is 0 , both $C_{g s 2}$ and $C_{q d 2}$ are shorted to VDD. As shown in Fig. 4(b), the RHP poles always exist. However, when $R_{g 2}$ is infinite, the RHP poles are moved to the LHP if $L_{d r v 1}$ is less than a threshold inductance $(0.6 \mathrm{nH}$ in our case). Hence, there is a threshold resistance for $R_{g 2}$ that can stabilize the PA if $L_{d r v 1}$ is small enough.

The evolutions of the RHP pole with positive imaginary part as a function of $R_{g 2}$ is shown in Fig. 5 for different $L_{d r v 1}$ values. The other conjugate pole with negative imag-
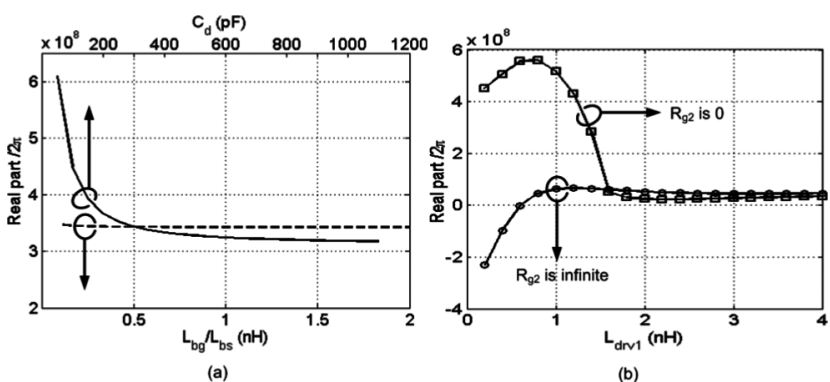

Fig. 4 (a) Evolution of the real parts of the RHP poles in $H_{c l, c m}$ as a function of $L_{b g}, L_{b s}$ and $C_{d}$; (b) The real part of the RHP poles as a function of $L_{d r v 1}$ when $R_{g 2}$ is 0 and infinite.

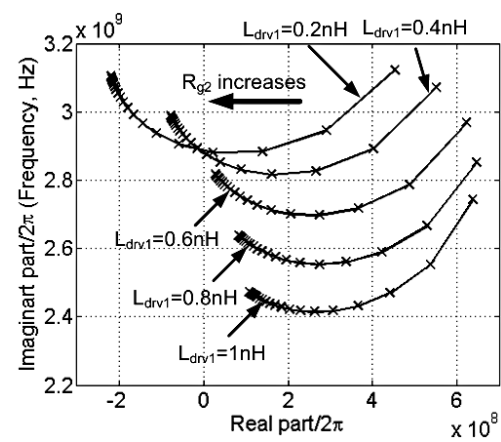

Fig. 5 Evolutions of the RHP pole with positive imaginary part in $H_{c l, c m}$ against $R_{g 2}$ for different $L_{d r v 1}$ values. 


$$
Y_{c m}=\left[\begin{array}{cccccc}
s\left(C_{d r v 1}+C_{g s 1}\right) & -s C_{g d 1} & 0 & 0 & -\frac{1}{s L_{d r v 1}} & -s\left(C_{d r v 1}+C_{g s 1}\right) \\
+s C_{g d 1}+\frac{1}{s L_{d r v 1}} & s\left(C_{g d 1}+C_{d s 1}+C_{g s 2}\right) & -s C_{d s 2} & -g_{m 2}-s C_{g s 2} & 0 & -g_{m 1}-s C_{d s 1} \\
g_{m 1}-s C_{g d 1} & +s C_{d s 2}+g_{m 2} & s\left(C_{d s 2}+C_{g d 2}\right) & g_{m 2}-s C_{g d 2} & -Y_{d} & 0 \\
0 & -g_{m 2}-s C_{d s 2} & +Y_{d} & s\left(C_{g s 2}+C_{g d 2}\right) & -\frac{1}{2 R_{g 2}} & 0 \\
0 & -s C_{g s 2} & -s C_{g d 2} & +\frac{1}{2 R_{g 2}} & \frac{1}{2 R_{g 2}}+Y_{d}+\frac{1}{s L_{d r v 1}} & -\frac{1}{2} s C_{d} \\
-\frac{1}{s L_{d r v 1}} & 0 & -Y_{d} & -\frac{1}{2 R_{g 2}} & +\frac{1}{2 s L_{b s}}+\frac{1}{2} s C_{d} & \frac{1}{2} s C_{d}+g_{m 1}+\frac{1}{2 s L_{b g}} \\
-s\left(C_{d r v 1}+C_{g s 1}\right) & -s C_{d s 1} & 0 & 0 & -\frac{1}{2} s C_{d} & +s\left(C_{g s 1}+C_{d r v 1}+C_{d s 1}\right)
\end{array}\right]
$$

inary part moves in the same direction. Other poles stay in the LHP when $R_{g 2}$ and $L_{d r v 1}$ are changing. As shown in Fig. 5, the RHP pole moves towards the LHP as $R_{g 2}$ increases. The minimum $R_{g 2}$ needed to move the RHP pole to the LHP increases as $L_{d r v 1}$ increases. When $L_{d r v 1}$ exceeds some threshold, the use of $R_{g 2}$ can not move the RHP poles to the LHP anymore. A small $L_{d r v 1}$ can easily be implemented by using a differential inductor. This will not affect the performance in differential mode while the differential inductance can be set to what is needed. So the use of $R_{g 2}$ is quite efficient to stabilize the circuit in common mode when a differential inductor is used in the driver stage. The $\mathrm{K}$ factor of a differential inductor can be more than 0.8 , which means that the common-mode inductance can be very small. The $\mathrm{K}$ factor of the differential inductor in our case is about 0.83 . The common-mode inductance is $0.25 \mathrm{nH}$, while the differential inductance is $2.7 \mathrm{nH}$. This means the commonmode stability problem can be solved by $R_{g 2}$.

\section{Simulation Results}

Verification of the stability method was performed on a $2.45 \mathrm{GHz}$ Class-E PA designed in a Silterra $0.18 \mu \mathrm{m} \mathrm{RF}$ CMOS process. Figure 6 shows the simulated and calculated minimum $R_{g 2}$ to stabilize the circuit as a function of $L_{d r v 1}$ for different $C_{d}$ values. As shown in Fig. 6, the calculation results correspond to the simulation results very well. With increasing $C_{d}$, the maximum allowable $L_{d r v 1}$ that can

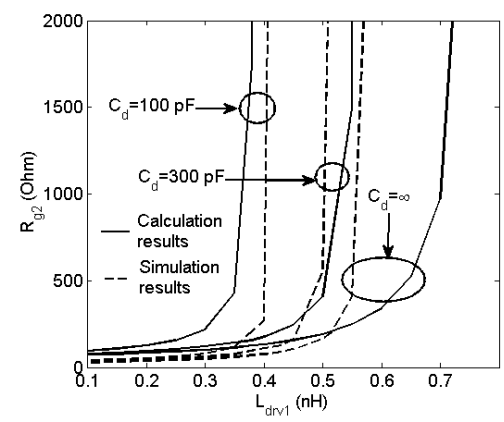

Fig. 6 Simulation and calculation results of the minimum $R_{g 2}$ to stabilize the PA as a function of $L_{d r v 1}$ for different $C_{d}$ values. be stabilized by $R_{g 2}$ is increasing. In our case, when $C_{d}$ is $100 \mathrm{pF}$, the maximum $L_{d r v 1}$ is around $0.4 \mathrm{nH}$ for both simulation and calculation results. This value becomes $0.6 \mathrm{nH}$ for simulation results and $0.75 \mathrm{nH}$ for calculation results when $C_{d}$ is infinite.

\section{Conclusion}

This paper proposes a new and practical common-mode stabilization method for a CMOS cascode Class-E PA with driver stage. By adding a resistor at the common-gate node of the cascode transistor, the PA is stabilized in common mode. The simulation results confirm the proposed stabilization method, showing that the proposed method is an effective way for the common-mode stabilization.

\section{Acknowledgment}

This work is supported by Essensium N.V. and by the Flemish Government under the IWT Grant 80334 ALOHA.

\section{References}

[1] J. Jugo, J. Portilla, A. Suarez, and J.M. Collantes, "Closed-loop stability analysis of microwave amplifiers," Electron. Lett., vol.37, no.4, pp.226-228, Feb. 2001.

[2] S. Jeon, A. SuareZ, and D.B. Rutledge, "Global stability analysis and stabilization of a class- $\mathrm{E} / \mathrm{F}$ amplifier with a distributed active transformer," IEEE Trans. Microw. Theory Tech., vol.53, no.12, pp.37123722, Dec. 2005

[3] C. Zhi, H. Yang, and R. Luo, "Novel stabilization method for eliminating oscillation in RF CMOS nonlinear power amplifiers," Proc. Int Conf., Communications, Circuits and Systems, vol.4, pp.2610-2613, 2006.

[4] M. Unterweissacher, K. Mertens, T. Brandtner, and W. Pribyl, "Stability analysis of on-chip multi-stage RF power amplifiers," IEE RFIC Sym., pp.471-474, 2007.

[5] D. Chowdhury, P. Reynaert, and A.M. Niknejad, "Transformercoupled power amplifier stability and power back-off analysis," IEEE Trans. Circuits Syst. II: Expr. Briefs, vol.55, no.6, pp.507-511, June 2008

[6] C. Yoo and Q. Huang, "A common-gate switched 0.9-W class-E power amplifier with $41 \%$ PAE in $0.25 \mu \mathrm{m}$ CMOS," IEEE J. SolidState Circuits, vol.36, no.5, pp.823-830, May 2001.

[7] K. Ogata, Modern control engineering, Prentice-Hall, NJ, 1997. 